\title{
CAPILLARY-GLIA RELATIONSHIPS IN THE HUMAN OPTIC NERVE, CHIASMA, AND TRACT, AS SHOWN BY PHASE-COLOUR CONTRAST AND MICRODISSECTION*
}

\author{
BY \\ C. A. ERSKINE \\ School of Anatomy, Trinity College, Dublin
}

In the optic nerve, chiasma, and tract the component most closely associated with the blood vessels is the neuroglia. It has become increasingly appreciated that in the brain and spinal cord the neuroglia has a dynamic role to play in metabolic processes, and is no longer to be regarded as primarily a supporting matrix; on the details of its association with the vascular system there is little agreement. As an extension of the central nervous system, the complex glial system in the optic nerve, chiasma, and tract can be expected to play an equally important part in function and pathology.

In view of the importance of the relationship between the vascular system, especially the capillaries, and the neuroglia and the metabolic activity of the neural elements in the central nervous system, an investigation was carried out by new methods to determine the distribution and relationships of the perivascular glia in normal human optic nerve, chiasma, and tract. The normal capillary-glia architecture in different regions of the optic system must be taken into account in instances of visual field defects which arise as the result of vascular disturbance or some other pathological condition. It is perhaps in the capillary-glia architecture that we might seek an explanation of certain differences in visual field defects which result from disturbances in the vascular system.

The literature on the glia of the optic system is remarkably scarce. Liss (1956) remarked that he could find only three reports on the astroglia in the optic nerve (Marchesani, 1926; Cone and Macmillan, 1932; Kolmer, cited by Lauber, 1936).

The astroglia are the elements of the neuroglia which have special relationships to the vascular system. Cone and Macmillan (1932) cited Marchesani's description of the astroglia in the optic nerve as shown by staining methods, and mentioned that the glia intervened in a typical manner between the vascular and nervous systems at the lamina scleralis. This followed the view of Achucarro (1918), who regarded this relationship as characteristic of the whole central nervous system. Cone and Macmillan (1932) stated that the neuroglial cells in the optic system did not differ in any way from those in the brain and spinal cord.

\footnotetext{
* Read at the combined meeting of the Society of British Neurological Surgeons and the 36th Annual Meeting of the Irish Ophthalmological Society in Dublin, May 16-18, 1957. 
Among the questions raised by a review of investigations on the blood vessels of the cortices of the brain and central tracts and the neuroglia are those which concern the existence of the glia membrane around the capillary endothelium, and if present its character and structure. How close is the relationship of the membrane or the glia cells and their processes to the capillary wall, and to the arteriole and venule adventitia? Does the glia differ in its degree of attachment to these different vessels in the nerve, chiasma, and tract? Can a potential space be demonstrated between the vessel wall and glial structures (the so-called space of His)? Further, does a subarachnoid extension (Virchow-Robin space) pass as far as the capillaries in the nerve, chiasma, and tract and thus intervene between the nutritive capillary and nerve elements? At least some of these questions may be answered by newer approaches to the problem of the glia vascular relationships.

\section{Material and Methods}

The material consisted of eight specimens of intracranial optic nerve, chiasma, and part of the optic tract behind the chiasma. These were obtained within 8 hours of death, chiefly in cases where this occurred suddenly by accident. In two instances the specimens were obtained in less than 2 hours in favourable conditions.

Frozen and paraffin sections were cut from the 48-hour formalin-fixed and bromformalin-fixed specimens. The sections varied in thickness from 1 to $5 \mu$. Control sections were stained with Cajal gold sublimate, del Rio Hortego silver carbonate, gallocyanin, and acid fuchsin. The sections for phase contrast-colour contrast microscopy were mounted unstained in glycerin jelly and glycerin and distilled water on slides and on cover slips. Examination of the sections was carried out with a new Leitz phase contrast-colour contrast condenser. Microdissection of the specimens was performed with a large sliding micromanipulator under optical phase and colouration. The instruments used were glass needles drawn to a tip diameter of approximately 8 and $20 \mu$.

The specimens were photographed during the operations, using Anscochrome 32 ASA film rated at 64 ASA and 100 ASA, and were developed accordingly.

\section{Normal Vascular-Glia Anatomy under Phase-Colour Contrast}

Attention was mainly directed to the capillary-glia relationships. The most favourable specimens were those fixed within 2 hours of death; changes in the cytological detail could be detected at 8 hours. All the illustrations reproduced below are taken from original $35-\mathrm{mm}$. colour transparencies.

Optic Nerve.-The relations of the capillaries contained in the pial septa were easily recognized (Fig. 1, opposite). The septa were seen to be covered by a neuroglial meshwork and vascular footplates. Capillaries between the nerve bundles which completely lack a connective-tissue covering could not be definitely identified. A glial meshwork was also clearly visible outside this thin connective tissue around an isolated capillary (Fig. 2, opposite). 


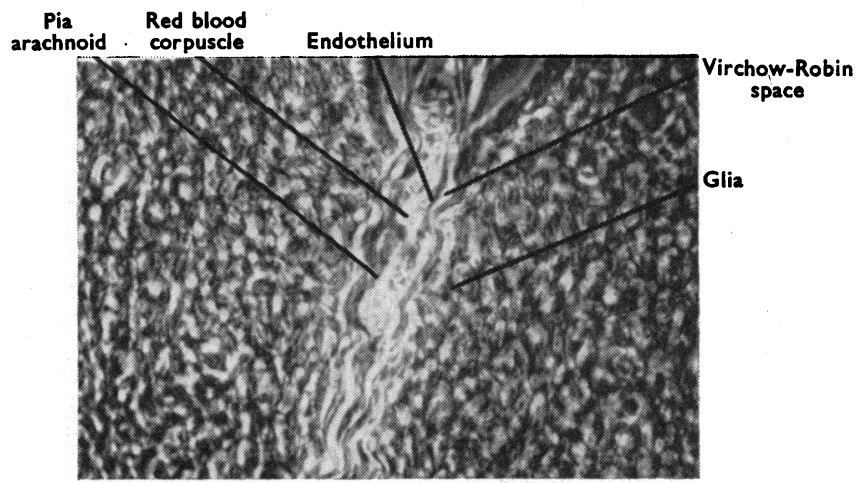

Fig. 1.-Optic nerve: pia-arachnoid extension in septal region; venous capillary visible in centre of connective tissue. $\times 400$.

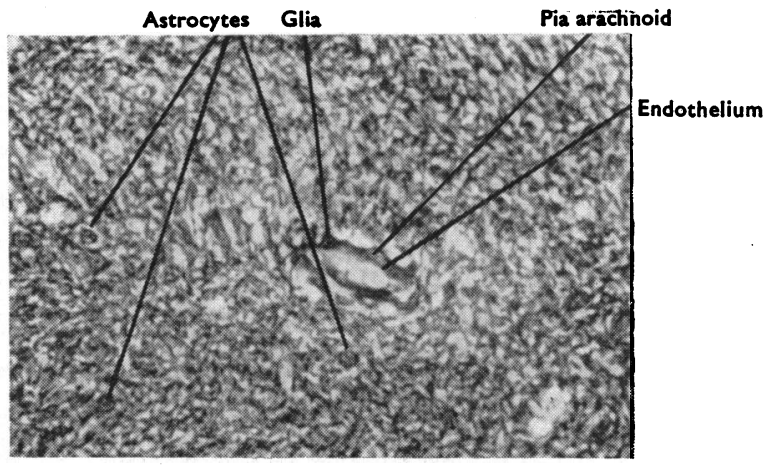

Fig. 2.-Optic nerve: segment of capillary with pia-arachnoid connective tissue between endothelium and glia. $\times 480$.

Chiasma and Tract.-In general the architecture of the chiasma is distinctive and different from that of the optic nerve. In particular most capillaries do not possess the pial covering which was seen to be characteristic of the nerve (Fig. 3). A glial mesh and vascular footplates can be

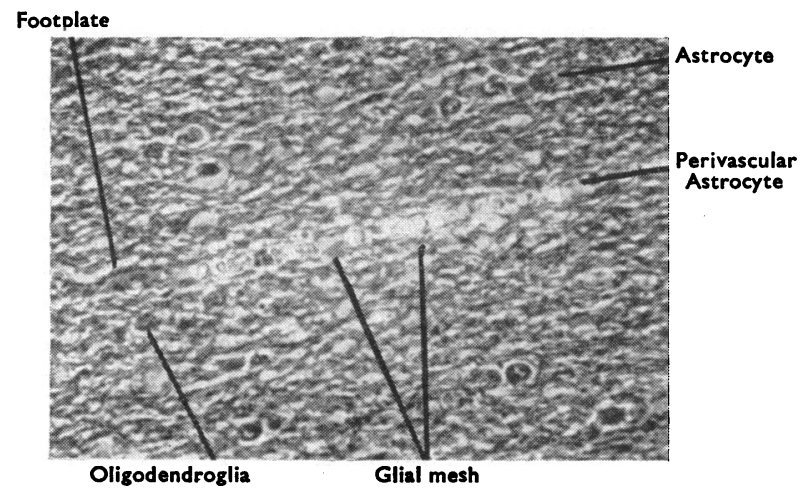

FIG. 3.-Chiasma: on the left the section passes above the roof of the capillary and the glial mesh and footplates are visible; sections of astrocyte footplates are also seen. Perivascular astrocytes are seen on the right. $\times 400$. 
distinguished in close relation to the smallest capillary. Satellite astrocytes and oligodendroglia cells could also be seen in characteristic dispositions.

In the tract the astrocytes (mainly fibrous in type according to their nuclear characteristics) and oligodendroglia were distinctive in their columnar arrangement characteristic of central tracts. Since the sections were thin (usually 2 or $5 \mu$ ) for phase, the processes could not be followed as far as those in thick-stained sections. The microglia could also be distinguished and in particular they did not show any pathological reactions; this was confirmed in the stained sections. As in the chiasma, the capillaries did not have the pial connective-tissue covering found in the nerve. These capillaries showed associated astrocytes and oligodendroglia, often arranged in groups as well as vascular footplates, in relation to the endothelium (Fig. 4).

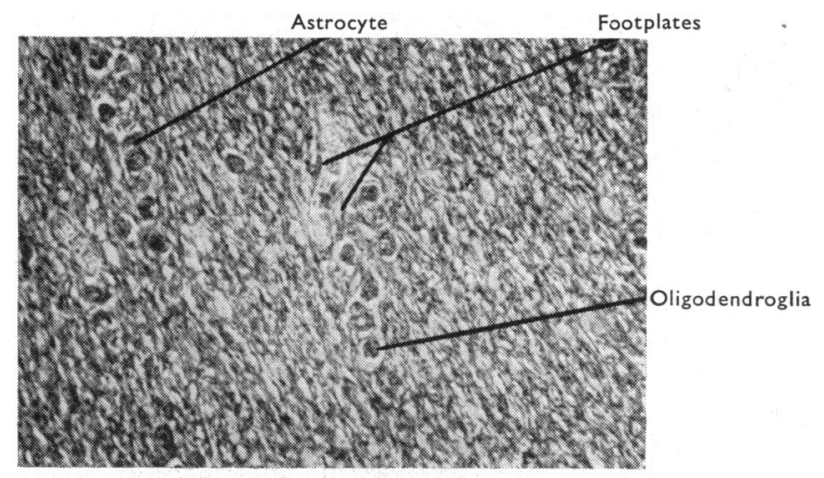

FIG. 4.-Optic tract: columnar and grouped astrocytes and oligodendroglia seen near capillary, and vascular footplates with fibrous processes on sides of endothelia. $\times 400$.

The continuity of the glia as a membrane was in doubt. Around the larger blood vessels the space of Held was easily distinguishable with the same characteristics as were revealed by stained sections; the smaller capillaries did not show a space of Held.

The phase-colour contrast method was a useful supplement to the classical staining techniques, since it simultaneously revealed cellular and architectural details of all the structures present. A disadvantage was the fact that thick sections could not be used.

\section{Normal Vascular-Glia Anatomy by Microdissection under Phase-COLOUR CONTRAST}

Care was taken to obviate artefacts in dissection by freeing the part of the section under examination from the slide before dissection.

Optic Nerve.-Since an apparent Virchow-Robin space was present around the vessels inside the pial septa by the phase method, a dissection of this space was carried out to determine its actual presence. Dissection revealed that the connective-tissue layers which bordered the space were not 
adherent to one another; it could be demonstrated that a Virchow-Robin space existed around the small vessels of capillary dimension in the septa.

The vessels outside the septa could not easily be freed from their connective tissue. Vascular footplates and a glial mesh were adherent to this perivascular connective tissue. Here a Virchow-Robin space could not be shown. This suggests that the Virchow-Robin space is closed and does not communicate with the potential space of His (as originally defined) around the capillaries.

\section{Chiasma and Tract}

(a) Precapillaries and Arterioles.-In posterior parts of the chiasma, where precapillaries and arterioles of about $40 \mu$ diameter can be found, it may be seen by phase-colour contrast that pial connective tissue is not present and perivascular glial footplates attach directly to the adventitia wall. By dissection, cross-section segments of these vessels could be freed and carried outside the nerve for examination (Figs 5 and 6, and Fig. 7, overleaf).
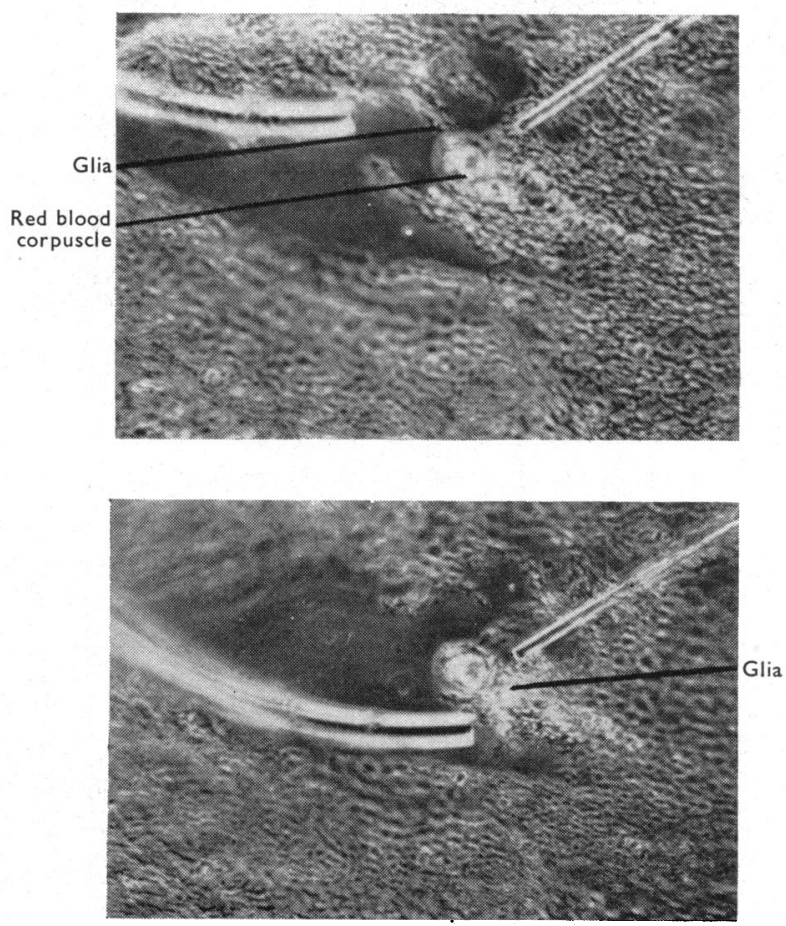

FIGS 5 and 6.-Chiasma: microdissection of precapillary; glial fibres adhere to vessel wall when segment is removed to exterior of section. $\times 240$.

The glial mesh was stripped from the vessel segment (Fig. 8, overleaf); this manipulation does not detach at least some of the vascular footplates from the adventitia. On cross-section they cannot be seen, but on turning the section on edge the remains become visible. 


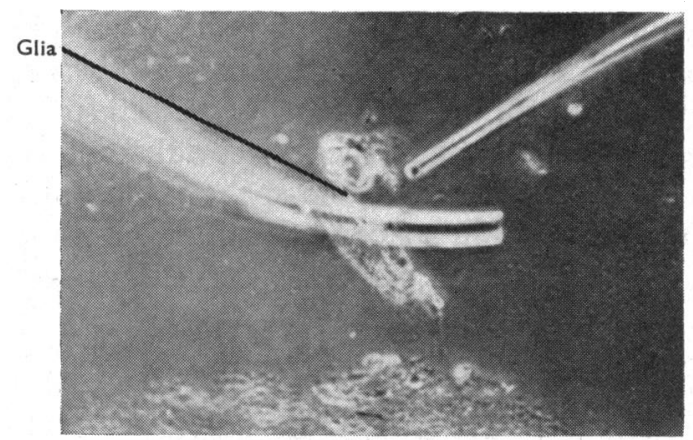

FIG. 7. - Chiasma: microdissection of precapillary; glial fibres adhere to vessel wall when segment is removed to exterior of section. $\times 240$.

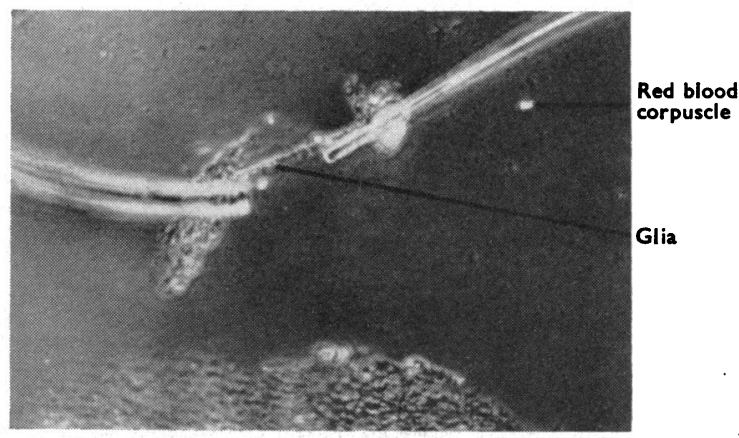

FIG. 8.-Chiasma: glial mesh stripped from precapillary wall (same vessel as in Figs 5, 6, 7). $\times 240$.

On a longitudinal section of a vessel of capillary dimensions which showed connective tissue as a component of its wall, a dissection demonstrated a similarly adherent glial meshwork (Fig. 9, and Figs 10, 11, 12, opposite).

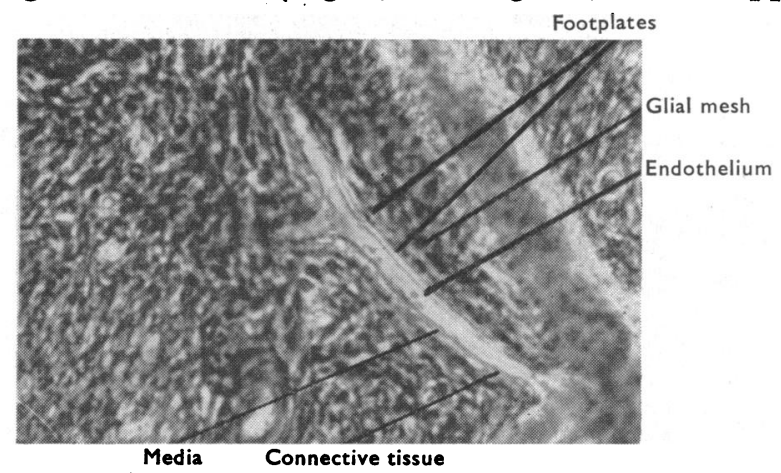

FIG. 9.-Chiasma: microdissection of arterial capillary; glial fibres attached to capillary wall remain adherent and resist stretching of wall. Diameter of needle-tip $8 \mu . \times 400$.

(b) Capillaries.-The capillary-glia relationships were examined by microdissection in the absence of a pial covering or connective tissue in the capillary wall (Fig. 13, overleaf). 

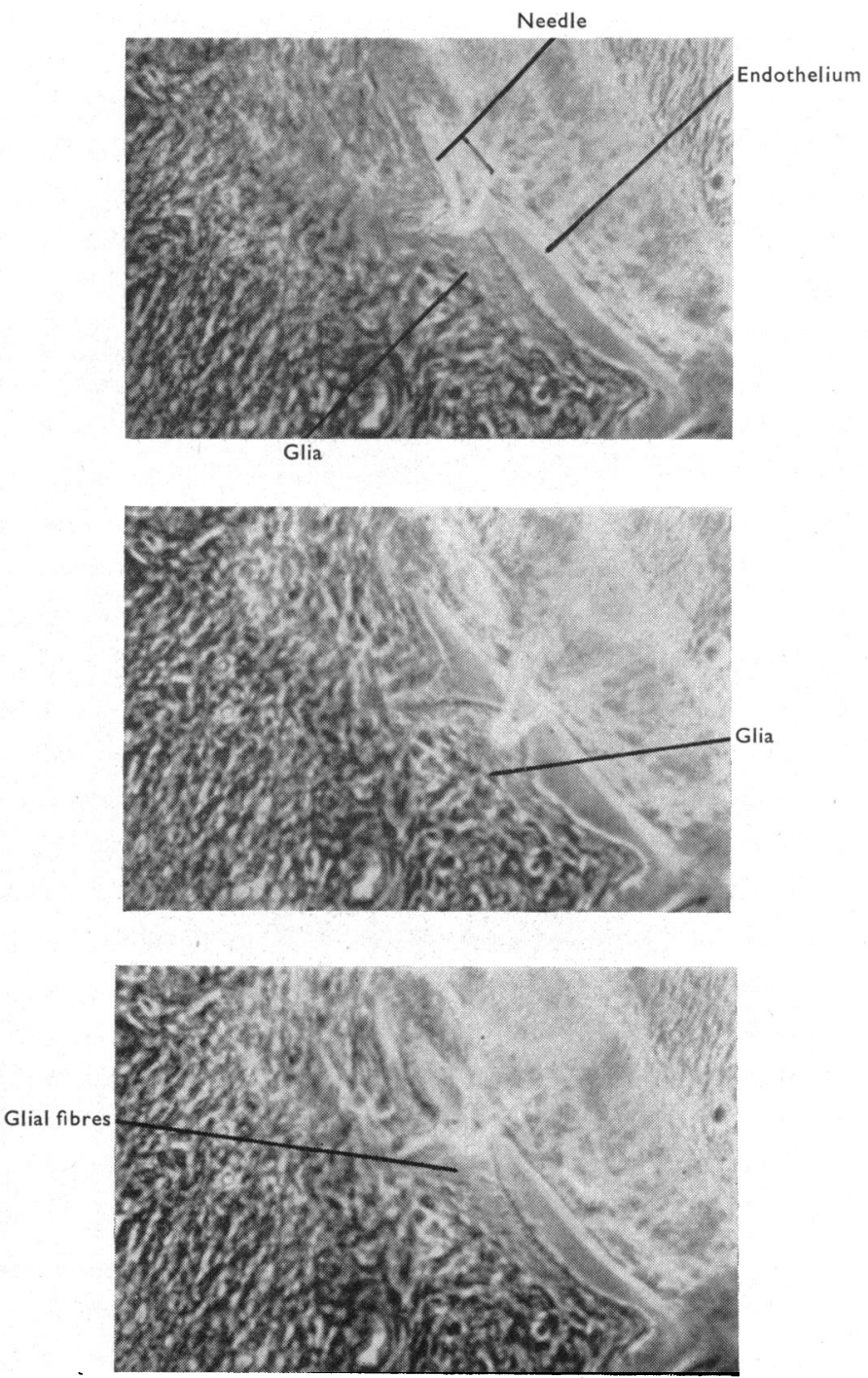

10, 11, 12.-Chiasma: microdissection of arterial capillary; glial fibres attached to capillary all remain adherent and resist stretching of wall. Diameter of needle-tip $8 \mu . \quad \times 400$.

a a longitudinal section, the capillary-glia network may be dissected to rmine the degree of attachment of the glia and its structure. This was : by drawing the glia away from the endothelium, where it was seen that wo structures did not adhere to one another.

is procedure demonstrated a space of His (Fig. 14, overleaf); the glia shown to consist of a fine mesh of sponge. At the capillary wall the 1 was seen to be bordered at least partly by a cuticle or membrane which not apparent before dissection. 


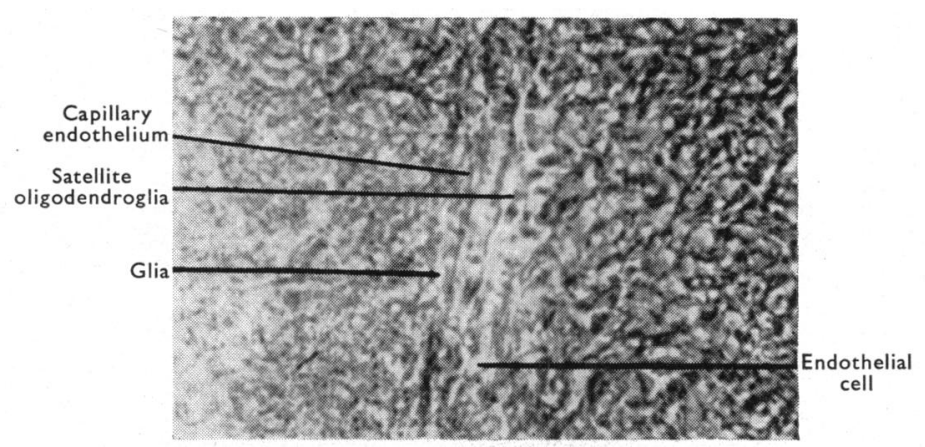

FIG. 13.-Optic tract: two capillaries seen close together without definite connective tissue outside endothelium; segments of glial processes ending in vascular footplates make connexion with endothelium. $\times 450$.

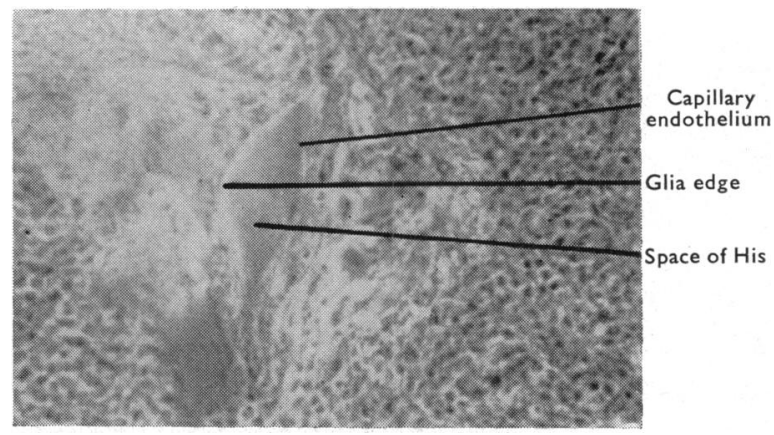

FIG. 14.-Optic tract: glia drawn away from endothelium and artefact space of His produced (same section as in Fig. 13). $\times 450$.

Thus it was seen that the glia was more closely attached to the vessels which possessed a definite adventitia and to pial connective tissue around the vessels than to the capillary where no connective-tissue layer could be seen.

\section{Discussion}

The relationship between the capillaries, the neuroglia, and the nerve tissue in the optic nerve, chiasma, and tract, might be expected to be similar to the relationships which are found in the brain and spinal cord. At the capillary level in the brain, Patek (1944) found that on the results of injection experiments no barrier between the vascular system and the nerve tissue existed. However, Glees (1955) found a definite delay in the transmission of injected dyes which must have its origin at capillary level, a finding supported by studies with radioactive isotopes. The situation in the optic nerve, chiasma, and tract was not discussed by Glees.

A close connexion between the oligodendroglia, astrocytes and their footplates, and the capillary wall has been assumed on the basis of staining methods. By microdissection under phase-colour contrast, it has been possible to demonstrate directly that the degree of attachment of the astrocytes and their processes to the blood vessels seems to depend on the presence 
of an adventitial layer. If adventitia is present the attachment is fixed, whereas if it is not present a cleavage space can easily be produced.

Glia intervened between the vascular and nervous tissue in the optic system, though a definite continuous membrane could not be shown in this instance; the glia intermediary consisted, apart from vascular footplates, of a fine mesh or sponge of glial fibres, bordered at least partly by a cuticle or membrane at the capillary level not clearly shown in staining techniques. The view of Patek (1944) that the so-called space of His in the brain between the glial mesh and the adventitia is erroneously described seems correct. It is doubtful if this potential space could be injected, since the glia is attached to the arteriole and venous adventitia; but around the capillaries where adventitia is absent and where the glial attachment is less fixed it would seem that an artefact space could be produced.

Connective tissue of pial origin intervened between the nerve elements and the capillaries in large parts of the optic nerve. Capillaries between the nerve fibres did not have a Virchow-Robin space around them. At the same time capillaries in the septal region of the optic nerve were surrounded by a Virchow-Robin space. Connective tissue was not found in most of the chiasma and tract, where the findings of François, Neetens, and Collette (1956) could be confirmed that there is a noticeably richer supply of capillaries. Here a Virchow-Robin space was not found at the capillary level (which agrees with the findings of Kuhlenbeck (1951) for the cerebral cortex). Thus the presence of a Virchow-Robin space seems to depend not so much on the size of the vessel as on the amount of pial connective tissue which is present around it.

The pattern described in the glia-vascular relationship raises further questions. Similar methods to those described could be used to investigate the changes in the glia-vascular pattern in experimental or pathological alterations in the circulation in the optic nerve, chiasma, and tract.

\section{SUMMARY}

Microdissection of fixed tissues combined with a new phase contrastcolour contrast optical system was used to investigate the capillary-glia relationships in the normal human optic nerve, chiasma, and tract. It was concluded that glial elements in the form of a mesh separate the nerve fibres from the capillary at every point examined; the presence of a glial membrane was uncertain. The presence of connective tissue around the endothelium seemed to determine the degree of fixation of the glial mesh; the glia was attached to adventitia, whereas in the absence of connective tissue the glia was not fixed to the capillary endothelium. A Virchow-Robin space could be clearly demonstrated in the septal region of the optic nerve in relation to capillaries, but not at the capillary level in the chiasma and tract where perivascular pial connective tissue was usually absent; therefore the presence 
of a Virchow-Robin space depended on the amount of related connective tissue and not on the size of the vessel.

\section{REFERENCES}

ACHUCARro, N. (1918). J. nerv. ment. Dis., 48, 333.

Cone, W., and MacMillan, J. A. (1932). In "Cytology and Cellular Pathology of the Nervous System", ed. W. Penfield, vol. 2, p. 839. Hoeber, New York.

François, J., Neetens, A., Collette, J. M. (1956). Brit. J. Ophthal., 40, 730.

GLEES, P. (1955). "Neuroglia: Morphology and Function". Blackwell, Oxford.

KOLMER, W., cited by LAUBER (1936). "Handbuch der mikroskopischen Anatomie des Menschen", ed. W. von Möllendorf, vol. 3, pt 2, pp. 468-493. Springer, Berlin.

KUHLENBECK, H. (1951). Anat. Rec., 109, 375.

Liss, L. (1956). J. comp. Neurol., 105, 151.

MARCHESANI, O. (1926). v. Graefes Arch. Ophthal., 117, 575.

PATeK, P. R. (1944). Anat. Rec., 88, 1. 